\title{
Fault Localization via Risk Modeling
}

\author{
Ramana Rao Kompella, Jennifer Yates, Albert Greenberg, and Alex C. Snoeren, Member, IEEE
}

\begin{abstract}
Internet backbone networks are under constant flux in order to keep up with demand and offer new features. The pace of change in technology often outstrips the pace of introduction of associated fault monitoring capabilities that are built into today's IP protocols and routers. Moreover, some of these new technologies cross networking layers, raising the potential for unanticipated interactions and service disruptions, which the individual layers' built-in monitoring capabilities may not detect. In these instances, operators typically employ higherlayer monitoring techniques such as end-to-end liveness probing to detect lower- or cross-layer failures, but lack tools to precisely determine where a detected failure may have occurred. In this paper, we evaluate the effectiveness of using risk modeling to translate high-level failure notifications into lower-layer root causes in two specific scenarios in a tier-1 ISP. We show that a simple greedy heuristic works with accuracy exceeding $80 \%$ for many failure scenarios in simulation, while delivering extremely high precision (greater than $80 \%$ ). We report our operational experience using risk modeling to isolate optical component and MPLS control-plane failures in an ISP backbone.
\end{abstract}

\section{INTRODUCTION}

$\mathbf{O}$ PERATIONAL backbone networks are intrinsically exposed to a wide variety of faults and impairments. The networks are large, geographically distributed, and constantly evolving with complex hardware and software artifacts. A typical tier-1 backbone network consists of over a thousand routers from different vendors, with disparate feature sets, acting in various roles in the network architecture, supported by access and core optical transport networks involving more than two orders of magnitude more network elements. Backbone networks are layered in an attempt to help contain complexity within simple well-defined abstractions; in practice, however, layering often gives rise to additional, complex failure modes involving interactions between the different layers.

A large fraction of any tier-1 provider's time is spent coping with operational failures. The essential problem of fault management is to detect, localize, mitigate and ultimately correct any condition that degrades network performance. To assist in fault management, many network elements (such as routers) are designed to continuously monitor certain behaviors (e.g., link connectivity) and raise an alarm in the event of failure. In some cases, the network element can even perform

Portions of this manuscript appeared in ACM/USENIX NSDI 2005 and IEEE Infocom 2007. This work was supported in part by the National Science Foundation through a NSF CAREER award (CNS-0347949).

R. R. Kompella is with the Department of Computer Science, Purdue University, West Lafayette, IN 47907 (e-mail: kompella@cs.purdue.edu). This work, however, was done when the author was at UCSD.

J. Yates is with AT\&T Labs - Research, Florham Park, NJ 07932 USA (email: jyates@research.att.com).

A. Greenberg is with Microsoft Research, Redmond, WA 98052 USA (email: albert@microsoft.com).

A. C. Snoeren is with the Department of Computer Science, University of California, San Diego, La Jolla, CA 92093 USA (e-mail: snoeren@cs.ucsd.edu). automatic recovery actions such as re-routing packets through other functional paths. In other cases, the alerts are transmitted to semi-automatic fault management systems [16], which assist a human operator in troubleshooting the failure. The challenge in diagnosing many problems is that alarms indicate only that a network element observed a deviation from normal behavior (e.g., link failure or probe packet loss); the actual fault could lie anywhere in the network (e.g., at a downstream router or optical amplifier). In other words, the element that issues an alert is not necessarily the cause of the failure. Moreover, faults in lower layers are frequently first reported by monitoring systems at a higher layer. Hence, cross-layer associations are critical for accurate localization.

In this paper, we consider the effectiveness of an automated fault localization framework based on risk modeling to identify possible network failure locations given a set of potentially related alarms. At a high level, risk modeling involves mapping the dependency relationships between observable events and potential causes. For example, a single fiber cut can simultaneously affect multiple IP links that are carried over the fiber; therefore, the fiber represents a shared risk for all the IP links that traverse it.

While we believe that the risk modeling framework itself may be generally applicable in other scenarios, the construction of a complete and accurate risk model is often extremely challenging, and in some cases may not even be possible. Thus, we study the performance of risk modeling in the context of a restricted class of faults for which we can indeed construct practical risk models. In particular, we apply risk modeling to two distinct problems - link and path fault localization - that have been observed in practice in ISP backbone networks. In each instance, we collect failure signatures from a fault detection system deployed in a tier-1 ISP and construct a topologydependent risk model. The signatures and risk models are then input to a localization algorithm that outputs a hypothesis corresponding to a set of likely faults in the network.

LINK FAULT: In the first scenario we study, an IP network is constructed with point-to-point links (optical circuits) between different routers on top of an underlying optical topology. Monitoring alarms associated with optical circuit failures are typically generated on an individual basis-for example, a router failure will appear as a failure of all links terminating at that router. Best current practice requires a manual correlation of the individual link failure notifications to determine that they are all because of a common network element (e.g., router). In more complicated failure scenarios, however, it is substantially more challenging to group individual alarms into common groups, and often difficult to even identify in which layer the fault occurred (e.g., in the transport network interconnecting routers, or in the routers themselves).

PATH FAULT: In the second scenario, we consider an MPLS 
network, where MPLS labels are established between two edge routers along the shortest path identified by the underlying interior gateway protocol (IGP) such as OSPF or IS-IS. Such an architecture is currently used by many tier-1 ISP networks. One commonly observed failure scenario occurs when OSPF re-routes due to a change in IGP link weight, but MPLS does not update its label-switched paths accordingly. Therefore, all the packets belonging to that particular MPLS tunnel attempt to traverse the old path only to be dropped within the network. Another common reason for such black holes involves configuration or operator error [9]. For example, a human may forget to enable MPLS on a newly added interface causing MPLS-switched packets to be dropped at that interface.

In each scenario, we evaluate the efficacy of a greedy localization heuristic using real failure data and risk models constructed from monitoring systems deployed on a tier1 backbone network. Our results show that a simple riskmodeling approach can deliver useful results even with imperfect dependency models and inaccurate failure data. While researchers have previously proposed sophisticated localization algorithms based on Bayesian inference [31] and belief propagation [34], we demonstrate that greedy heuristics are both reasonably accurate (over $80 \%$ in our evaluation) and robust to the noise and errors found in real alarm and dependency data. The system described here is now in operational use at a tier-1 provider.

The remainder of this paper is organized as follows. We introduce the risk-modeling approach, localization algorithms and system architecture in Sections II, III, and IV respectively. We then present simulation results and experience with real data for both the systems in Section V We present a brief discussion on insights that we have learnt through our experience in SectionVI. Finally, we present related work in Section VII before concluding in Section VIII.

\section{Shared RISK ANALYSIS}

The key idea for localization of the two failure scenarios mentioned earlier is shared risk analysis. Roughly speaking, a physical object such as a fiber or an optical amplifier represents a shared risk for a group of logical entities at an upper layer such as the IP layer. That is, if the optical device fails or degrades, all of the IP components that rely upon that object fail or degrade. Similarly, a link between two routers can form a shared risk for multiple MPLS tunnels (or end-to-end paths) that pass through that link. In the literature, such associations are referred to as Shared Risk Link Groups or SRLGs [6]. This concept is well understood in the context of network planning where backup paths are chosen such that they do not have any SRLGs in common with the primary path, and sufficient capacity is planned to survive SRLG failures. The application of risk group models to real-time and offline fault analysis, however, has yet to be explored.

In typical failure scenarios, network operators often require an accurate cross-layer view of the network to localize the root cause. Thus, in many ISPs, fault localization is performed at a centralized location where the required cross layer as-
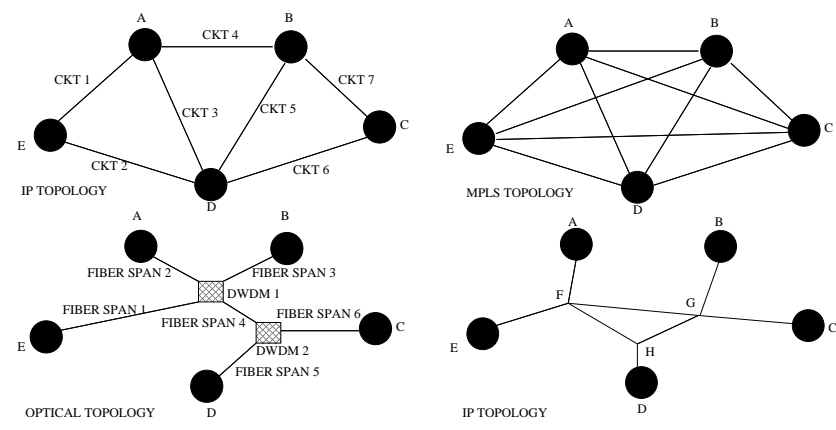

(a) IP-optical topology for LINK (b) MPLS topology for PATH FAULT FAULT

Fig. 1. Example of SRLGs for the two example failure scenarios considered in the paper.

sociations are easily constructed. Of course, the fault notifications themselves are generated by the various devices and components distributed throughout the network. Within the centralized location, however, several (possibly independent) fault localization systems, each of which is customized to a specific category of faults, often exist. The specialized nature of these tools is due primarily to the existence of a wide variety of faults both within as well as across layers, thus making it difficult to create one integrated platform for all types of faults. For example, in LINK and PATH FAULTS, the root causes are optical component and IP interface failures, respectively; creating a unified heterogeneous system would not only be difficult but in fact unnecessary. In this paper, therefore, we consider the two problems-LINK and PATH FAULTS-in isolation, despite applying the same risk-modeling framework.

Shared risks in LINK FAULT: We construct a model of risks that represent the set of IP links that are likely to be affected by the failure of each component within the network. The basic IP network topology can be represented as a set of nodes interconnected via links. Inter-domain and intra-domain routing protocols such as OSPF and BGP operate with a basic abstraction of a point-to-point link between two routers. These IP links in turn are overlaid on top of an underlying optical network. Despite appearing logically disparate, multiple IP links can share components in the optical network, thus leading to shared risks in the network.

In many tier-1 backbone networks, each inter-office IP link is carried on an optical circuit (typically using SONET). This optical circuit in turn consists of a series of one or more fibers, optical amplifiers, SONET rings, intelligent optical mesh networks and/or Dense Wavelength Division Multiplexing (DWDM) systems [27]. These systems consist of network elements that provide O-E-O (optical to electrical to optical) conversion and, in the case of SONET rings or mesh optical networks, protection/restoration to recover from optical layer failures. Multiple optical fibers are then carried in a single conduit, commonly known as a fiber span. Typically, each optical component may carry multiple IP links-the failure of a single physical component results in the failure of all of these IP links. Therefore, physical elements such as fibers, fiber spans, and optical components constitute shared risks. In addition to the optical components, our SRLG model also 
includes routers (a shared risk for the set of links originating at the router), modules (common to the set of links connected to a particular module within a router) and ports (relied upon by the interfaces themselves). We also extend beyond physical components and include logical software entities such as OSPF area, router software version, etc., in the list of SRLGs.

We illustrate this concept in Figure 1(a) with a simple network consisting of five nodes connected via seven logical IP links (circuits CKT 1 through 7 shown in upper half of Figure 1(a)). These IP links are routed over shared optical layer components shown in the bottom half of Figure 1(a). These shared risks in the optical layer are denoted as FIBER SPAN 1 to 6, DWDM 1 and 2. CKT3 and CKT5 are both routed over FIBER SPAN 4 and thus would both fail with the failure of FIBER SPAN 4. Similarly DWDM 1 is shared between CKT 1, 3, 4 and 5, while CKT 6 and CKT 7 share DWDM 2.

Shared risks in PATH FAULT: In this problem, MPLS tunnels share individual IP links and hence form the shared risks. Each individual link failure or degradation can simultaneously affect all the MPLS tunnels that ride through that particular link. In fact, extending it a bit further, if there are multiple VPNs that ride through a given MPLS tunnel that rides through a failed link, all these VPNs are affected by a failure on that link. However, for the purposes of this paper, we focus on the failure of MPLS tunnels, while noting that we can extend this arbitrarily into higher layers if need be.

For example, in Figure 1(b), edge nodes A through E are connected via intermediate nodes $\mathrm{F}$ through $\mathrm{H}$. Clearly, from the figure, we can observe that the set of paths A-F-G-B, A-FG-C, E-F-G-C, E-F-G-B (and the corresponding reverse paths) share a common link-the link from $F$ to $G$. Hence this link F-G forms a shared risk for this group of paths. Note that we do not include all the optical components and other SRLGs we model for LINK FAULT in this context as MPLS failures do not involve optical components.

\section{FAULT LOCALIZATION VIA RISK MODELING}

The previous section describes two instances where a given shared risk failure affects all the entities that are dependent upon it. Here, we use these risk models to develop a fault localization methodology that can aid network operators in troubleshooting failures in an automated fashion. Our approach has three main components-failure detection and risk modeling, the core localization algorithm, and a set of refinements to handle domain-specific imperfections. Failures detected at a higher layer (such as failed IP links or MPLS tunnels) are fed into a localization engine that spatially correlates these failures according to the risk model (based on the underlying topology) to identify a small set of likely locations of the failure. This localization step is the primary reconnaissance to a final-and often (necessarily) manual-step of actually diagnosing the root cause of the failure and fixing the problem.

\section{A. Failure detection and risk modeling}

One of the two main inputs to a localization engine is the failure signature. The failure signature consists of a set of

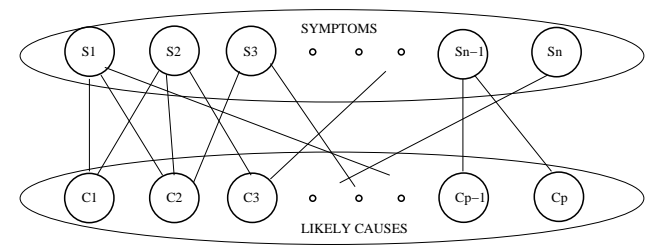

Fig. 2. Bipartite graph modeling of the failure localization problem as done in SCORE system.

symptoms observed due to a given failure. The symptoms themselves are typically detected through explicit monitoring (either through probes or lower-layer alarms). For example, in order to check IP link integrity, each router in the network injects periodic "HELLO" messages to the router at the other end, which then acknowledges the receipt of the message [22]. We consider the set of IP link failures that are temporally correlated (occur almost simultaneously) to constitute the failure signature in this scenario. Similarly, in monitoring MPLS tunnels for black holes, a monitoring server typically establishes connections (using, say, GRE tunnels [12]) with each edge router and injects periodic probes to every other edge router in the network and reports if any of the probes are lost. Therefore, the set of origin/destination pairs (ODpairs) that have lost connectivity (based on dropped probes) constitutes the failure signature in this scenario.

The other input required for fault localization is the risk model. We use a bipartite graph to represent the dependency between possible observable symptoms and corresponding likely causes as shown in Figure 2. An edge exists between a symptom and a likely cause if that symptom can be observed given a failure in that root cause. As shown in Figure 2, the top partition consists of the universal set of symptoms in the failure signature, and the bottom partition consists of the likely causes. We refer to the symptoms that have been reported by the failure detection system as observations. In LINK FAULT, the symptoms are IP link failures and the likely root causes are the SRLGs such as fiber spans, optical amplifiers, etc., while in PATH FAULT, observations are failed MPLS tunnels, while the underlying IP links make up the potential root causes.

\section{B. Fault localization}

Once we create these risk models, we then apply spatial correlation for fault localization. The observations that make up the failure signature are intersected according to the risk model to identify common shared risks. These shared risks form the most likely explanation for the failure signature; hence, the localization engine outputs this set of shared risks as the hypothesis. For example, in our LINK FAULT example in Figure 1(a), if CKT1 and CKT4 both fail simultaneously, it is highly likely that the failure will be in the optical component DWDM1 since this is the only shared risk among these circuits (or IP links). Similarly, in our PATH FAULT example in Figure 1(b), if the set of paths A-F-G-B, A-F-G-C, E-FG-C, E-F-G-B all fail in a given time interval (temporally correlated failures), spatial correlation leads to the only link that is common to all these paths-the link from F to G. The 


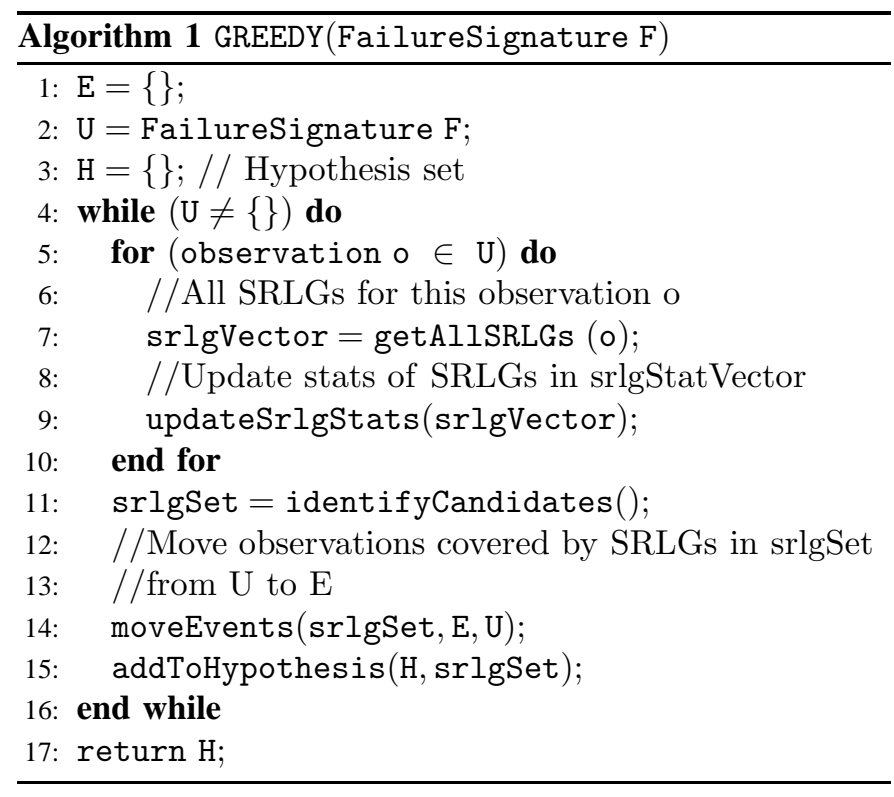

localization engine would, therefore, return the singleton set $\{\mathrm{F}-\mathrm{G}\}$ as the hypothesis in this failure scenario.

In most situations, however, it is not as straightforward to localize failure(s) as in the above toy examples. The localization engine does not have access to an oracle to determine whether a single failure, dual failure, or multiple failures occurred in the network; it has to determine the cardinality of the set of failures based on the failure signature alone. For any given failure signature, there could be many different likely explanations. Exploring all the possibilities is often not feasible. Such enumeration is also likely to contain many cases that are not typically observed in practice. We therefore attempt to identify hypotheses that can explain a given failure signature with the smallest set of failures. Our approach is in accordance with the principle of Occam's razor, that suggests that the simplest explanation is most likely. In order to find the simplest explanation (the one that hypothesizes least number of failures), an obvious strategy is to find the minimum set cover for the bipartite graph. Each shared risk is associated with a set of observations according to the risk model. While the general set-cover problem is NP-hard, the greedy approximation finds a solution guaranteed to be an $O(\log n)$-approximation to the optimal [18].

We use the core algorithm GREEDY (shown in Algorithm 1) to iteratively pick SRLGs that are the best according to a metric that we define later; updateSrlgStats() computes this metric for all SRLGs and identifyCandidates() picks the best SRLGs in the current iteration. It then prunes the set of observations explained by these candidate SRLGs (in moveEvents()) from the failure signature and repeats the process until no more observations remain in the failure signature. In the classic set-cover problem, the metric is the number of new observations covered by the candidate SRLG. In other words, in each iteration, GREEDY just picks those SRLGs that have the largest number of edges in the bipartite graph among those in the failure signature. For now, we deliberately abstract the identifyCandidates() and updateSrlgStats() routines since the metric of interest can change according to the particular problem at hand. In the next subsection, we discuss suitable choices for the two problems we consider.

\section{Managing imperfections}

In practice, the effectiveness of the greedy approach is limited by noise and incompleteness in the failure signature and churn in the risk models. We explain how the algorithm can be adapted to accommodate these imperfections in this section.

1) Imperfections in failure signature and risk model: In most real networks, there is constant churn due to various types of routing, congestion, maintenance and other related events. Due to this noise, there could be spurious observations in the failure signature that are not directly related to the particular real failures that we seek to localize, thus complicating localization. Besides noise, inherent inefficiencies in the detection system or losses of failure notifications due to unreliable transport mechanisms could lead to an incomplete failure signature. The extent of both these imperfections varies based on the particular domain. As with the failure signature, there are likely to be imperfections in the risk model as well. These imperfections also stem from inherent churn in the network, either due to out-dated databases or an inability to obtain exact dependencies. Similar to the failure signature, the extent of these imperfections depends upon the particular failure domain under consideration. We explore both imperfections in the context of the two problem domains we consider below: LINK FAULT suffers from relatively few imperfections, while PATH FAULT has many more sources of noise.

LINK FAULT. The detection mechanism in LINK FAULT uses both lower-layer alarms (such as a loss-of-signal alarm) and high-frequency, router-to-router probes (such as OSPF Hello messages) to detect losses in connectivity. Therefore, detection of IP link-layer faults is typically complete, in the sense that all IP links that fail due to a problem in the underlying optical network are detected with extremely high probability. A few fault messages are sporadically dropped during transmission, however, leading to a slightly incomplete failure signature.

The optical topology from which the risk model is computed is more or less static ${ }^{1}$, suggesting that the risk model would be easy to capture. In our experience, however, the risk models for the large majority of optical networks are maintained through human-entered databases that may drift away from reality over time leading to occasional errors in the database. These errors can affect the localization results and must therefore be factored into the localization algorithm.

PATH FAULT. The detection mechanism employed in PATH FAULT, on the other hand, consists primarily of end-to-end probes that operate at a much lower frequency. So, for many reasonable failures, the failure signature is not complete. Of course, if the failure persists, eventually all low frequency probes that go through the failure are bound to detect the failure. But this means that we need to wait for a long time

\footnotetext{
${ }^{1}$ Some optical networks perform lower layer re-routes to mask higher layer failures, in which case, paths need to be dynamically computed. We ignore this detail for the purposes of this paper.
} 
until the failure signature is complete. Furthermore, because the topology is large (on the order of a million paths), any failure can affect a significant number of paths leading to an overwhelming number of observations and a large failure signature set. In order to operate in real time, the failure signature must occasionally be down-sampled. Therefore, in this domain, the failure signature is expected to be largely incomplete. In addition to the incomplete failure signature, noise can cause some probes to be dropped in the network thus adding spurious observations to the failure signature.

The risk model in PATH FAULT for MPLS paths is primarily derived from the underlying IP topology. IP topology is inherently subject to churn due to various routing changes caused during failures, congestion and maintenance activities. Therefore, the risk model needs to be computed on-the-fly for this scenario from the topology snapshots during the failure interval. Because the topology is extremely large, it is often impractical to construct the entire risk model; the fault localization algorithm needs to be designed to operate with a partial risk model.

2) Handling imperfections through relaxed metrics: In order to handle these imperfections, we use two different metrics to identify the candidate SRLGs in each iteration of the basic GREEDY algorithm. Let $G_{i}$ correspond to the $i$ th shared risk in the network and $\left|G_{i}\right|$ be the total number of observations that belong to the SRLG $G_{i} \cdot\left|G_{i} \cap F\right|$ is the number of elements of $G_{i}$ that also belong to $F$, the failure signature. We define hit ratio of the group $G_{i}$ as $\left|G_{i} \cap F\right| /\left|G_{i}\right|$. In other words, the hit ratio of a group is the fraction of elements in the group that are part of the failure signature. The coverage ratio of a group $G_{i}$ is defined as $\left|G_{i} \cap F\right| /|F|$, i.e., the fraction of the observation explained by a given risk group.

If we have access to the complete failure signature and an accurate risk model, we can exploit the fact that every failed shared risk would have all associated observations in the failure signature. In other words, the hit ratio for the failed shared risks should be 1, so an optimal algorithm would select SRLGs with the highest coverage among those with a hit ratio of 1 . In the LINK FAULT, however, while we potentially have access to the entire failure signature and risk model, they are likely to contain a relatively small number of errors. In order to account for these errors, we define the SCORE algorithm [19] that considers SRLGs with a hit ratio greater than a particular error threshold, which is generally slightly less than 1 . We explain later in Section IV-C how we determine the error threshold in practice. Thus, in SCORE, the updateSrlgStats() routine computes the hit and coverage ratios, while identifyCandidates() routine picks the SRLG with the highest coverage ratio among those with hit ratio greater than the threshold.

On the other hand, if we do not have access to the entire failure signature or associated risk model, we cannot compute a meaningful hit ratio for each and every SRLG in the first place. Therefore, in such situations, the greedy heuristic picks those SRLGs that have the highest coverage-ratio in every iteration to output the hypothesis. In the resulting algorithm MAX-COVERAGE, the identifyCandidates () routine is similar to that of SCORE algorithm, except the condition on the hit ratio is eliminated. Since the hit ratio is not required, the updateSrlgStats() routine does not need to compute it as well. This algorithm, therefore, is applicable to PATH FAULT where we can compute the coverage ratios of all the SRLGs but not the hit ratios.

3) Additional refinements: While our relaxed selection metrics allow the localization algorithm to adapt to various operational realities, our localization architecture supports further domain-specific refinements. In particular, the input to as well as the output from the localization algorithms can be post-processed in order to better accommodate the needs of the particular domain. For instance, we introduced an error threshold above to deal with the slight possibility of errors in the risk model for LINK FAULT; the choice of error threshold, however, is not immediately clear. Another problem is that the output may still contain several root causes, some of which exist because the algorithm is trying to explain each and every symptom that is input to the system. This situation arises frequently in the PATH FAULT scenario; hence, we seek to output only the most significant root causes by effectively denoising the hypothesis. Another problem specific to the PATH FAULT domain is the need to consider multiple risk-models as the fault can be result of either the old or new topologies. We describe refinements to address these issues in Section IV-C.

\section{SYSTEM ARCHITECTURE}

So far, we have described the three required ingredients for fault localization - a failure detection system, a risk model, and the localization algorithm. In this section, we elaborate on how these components are instantiated to address the LINK and PATH FAULTS problems.

\section{A. Failure detection}

The failure signature upon which spatial correlation is applied is obtained from various types of network monitoring data sources depending on the particular failure scenario. In any case, we assume a monitoring system continuously generates a raw event stream (as shown on the left in Figure 3) with associated timestamps. For link faults, we use router syslogs generated by the router when it detects a link to be down. In contrast, path failure notifications are generated in response to the loss of end-to-end probes that constantly poll connectivity between various origin-destination pairs.

Data sources based on discrete asynchronous events, such as router syslog messages, need to be clustered to identify a failure signature. Note that a failure can cause symptoms that appear slightly off in time either due to time-synchronization errors across various elements, or propagation delays in event recording. There are many different ways to cluster events. In LINK FAULT, we use a clustering algorithm based on gaps between failure events. We consider the largest chain of events that are spaced apart by a set threshold (called a quiet period) as potentially correlated events. The intuition is that two events that occur within a time period less than a given threshold (we use 30 seconds in our system) can be attributed to the same failure. In contrast, for PATH FAULT, due to the presence of an excessive number of events attributable to noise in the network, 


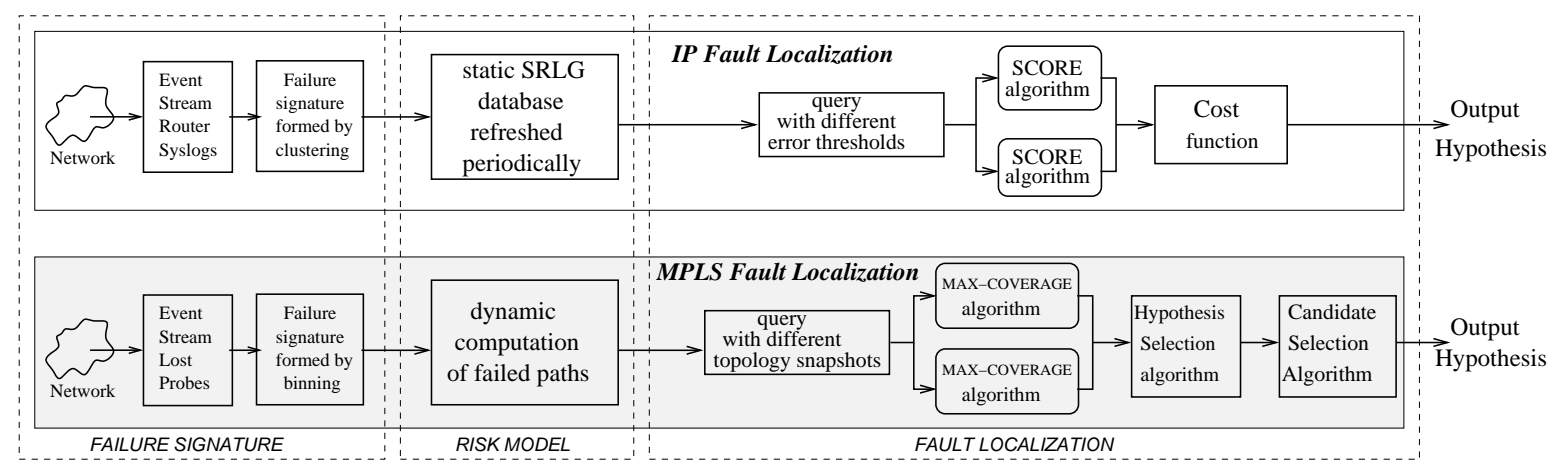

Fig. 3. System flow diagram for IP and MPLS fault localization problems.

a clustering scheme such as above results in clustering all events together into one cluster. Instead, we divide time into 15-minute bins. The particular choice of the bin size is somewhat arbitrary and can be tuned in accordance with the typical duration of a failure event, required timeliness of diagnosis, and amount of evidence that needs to be collected.

\section{B. Risk model}

In the middle portion of Figure 3, we show how the risk model is constructed for the two different failure scenarios. In LINK FAULT, the risk model is constructed from disparate databases that ISPs typically maintain for different types of shared risks. For example, optical layer shared-risks such as the SONET components that particular IP links traverse are extracted from databases populated from operational optical element management systems. Other risk groups such as OSPF area, router modules, etc., are populated by periodically polling configurations from the various network elements. The underlying databases track the network and therefore exhibit churn. We cope with database churn by regenerating risk groups multiple times during the course of the day.

For PATH FAULT, we construct the risk model dynamically from IP topology snapshots obtained through an OSPF monitor [29]. Because a significant number of failures in the MPLS domain are due to topology changes, we need to consider the topology snapshots both before and after the failure depending on the exact nature of the failure. Due to the dynamic nature of the risk model, we do not construct the entire topology for every snapshot, but instead obtain only the paths for the OD-pairs in the failure signature. Since there could be multiple paths between a given OD-pair due to equal-cost multi-path routing [14], we place the OD-pair in the risk groups corresponding to all the links that lie on at least one shortest path between the OD-pair.

\section{Localization algorithm}

Finally, once the failure signature is obtained and the risk model is constructed, we can perform fault localization as shown in the right portion of Figure 3. We proposed core algorithms for IP and MPLS fault localization in Section III-B; here, we discuss additional steps of processing required on top of the core algorithms for each of these systems as discussed in Section III-C.3.
LINK FAULT: Rather than fixing one particular error threshold for the system, we query the SCORE algorithm with multiple error thresholds (reducing from 1.0 to say 0.5) to obtain many different hypotheses as shown in Figure 3. The hypotheses obtained using different relaxations are then evaluated based on a cost function that depends on the error threshold and the size of the hypothesis. We use the ratio between the size of the hypothesis and the threshold as the cost; we seek to identify cases where a small relaxation in the threshold (an error threshold of 0.9, say) can reduce significantly the number of groups in the final hypothesis.

PATH FAULT: We use the MAX-COVERAGE algorithm that iteratively selects the links covering the most observations in the failure signature. As noted before, there are two issues we need to address. First, there can be potentially many different topology snapshots within a given failure interval and the question is which topology to use. To address this, we first generate multiple hypotheses for a given failure signature using all the available topology snapshots in the failure interval, and use a hypothesis selection algorithm, called UNION, that outputs the union of hypotheses generated with each of the available topology snapshots. By considering all possibilities, there is no loss in accuracy compared to an oracle that knows the ground-truth (and hence knows the right topology to pick), but precision is slightly lower.

Second, recall that the localization algorithm adds suspect links to the hypothesis until the hypothesis completely explains all the failed probes, including those observations that arise from inherent noise in the network. To address this, we use a candidate selection algorithm (called ABSOLUTE) that removes candidate links from the hypothesis that explain fewer than a threshold number of observations and thus focuses only on the main links in the hypothesis.

\section{Implementation}

For both the systems, we implemented the main localization algorithms in $\mathrm{C} / \mathrm{C}++$ and the rest in Perl. We also implemented a Web-based user interface for both systems. The IP fault localization system contains slightly more than 1,000 lines of $\mathrm{C}$ and about 2,500 lines of Perl code, while the MPLS fault localization system consists of about 5,000 lines of $\mathrm{C}++$ and about 2,000 lines of Perl code. 


\section{Evaluation}

In this section we evaluate the performance of systems we deployed to address both the LINK and PATH FAULTS problems in a tier-1 ISP backbone, using simulations as well as real network failure data. Before describing our results, we first present our metrics of evaluation.

\section{A. Metrics for comparison}

Ideally, we seek to evaluate the effectiveness of our algorithms by comparing their output to the ground-truth for the failures, i.e., the real root causes. We define two primary metrics for comparing our hypothesis with the ground-truthaccuracy and precision.

Accuracy is the fraction of elements in ground truth $G$ also contained in the hypothesis $H$, or $|G \cap H| /|G|$. If $G$ is a proper subset of $H$, then the accuracy is 1 . Any false negativesi.e., excluding a failed component from the hypothesis-will result in lower accuracy. This metric alone cannot capture the efficacy of the localization algorithm, however. For example, if we design an algorithm that always outputs $U$ where $U$ is the universal set of elements, then $G \subseteq U$ by definition, thus always leading to an accuracy of 1 . Such an algorithm obviously is not very useful in practice, however, due to its high false positive rate.

Therefore, we define precision to quantify the size of the hypothesis in relation to the ground truth. It is defined as the fraction of elements in the hypothesis that are also present in the ground-truth or $|G \cap H| /|H|$. In effect, precision captures the amount of truth in the hypothesis. For example, a precision of 0.9 would imply that the $90 \%$ of the elements in the hypothesis match the ground truth. Precision is roughly inversely proportional to false-positive rate: 0.9 precision is equivalent to $(1-0.9) / 0.9=11 \%$ false positives.

Typically, most algorithms tend to trade one metric for the other depending on how conservative or aggressive the algorithm is. A conservative algorithm tends to include all the possibilities in order to achieve better accuracy while losing precision, while an aggressive algorithm includes only the significant ones thus gaining precision while somewhat sacrificing accuracy. Our goal is to ensure that both these metrics are within reasonable bounds. While false negatives and false positives can also be chosen in place of accuracy and precision, we chose them since they represent the usability of our system more intuitively.

While precision provides one metric of conciseness, it is often difficult to interpret given the vast difference in the size of the true fault set (often one or two components) and the entire network of components. Hence, we also quantify the ability of our system to identify a small set of candidate faults using a metric we call localization efficiency. Localization efficiency is defined as the ratio of the number of suspect root-causes after localization to the number before. In other words, it is the fraction of root causes that are identified by our localization algorithm that likely explains a particular fault out of all the root causes that could cause a given fault. This metric is particularly useful in the context of LINK FAULT since it quantifies the ability of our tool to reduce the number of physical components that an operator needs to manually inspect. Define $G_{i}=\left\{g_{i 1}, g_{i 2}, \cdots, g_{i n}\right\}$ as the set of shared risks that a symptom $c_{i}$ depends on. Let $F$ denote the failure signature consisting of symptoms $\left\{c_{j 1}, c_{j 2}, \cdots, c_{j m}\right\}$ and $H$ be the best hypothesis for $F$. Clearly, $H$ is a subset of $U=\cup_{k=1}^{m} G_{j k}$, the union of all possible root causes. The localization efficiency is given by $|H| /|U|$.

\section{B. Results for LINK FAULT}

We begin by evaluating the accuracy of the SCORE algorithm for IP fault localization within a controlled environment by using emulated faults. We used an SRLG database constructed from the network topology and configuration data of a tier-1 service provider's backbone. We injected varying numbers of simultaneous faults and studied the efficacy of the algorithm in the presence of database errors and lossy fault notifications.

1) Algorithm accuracy: We simulated multiple simultaneous failures by picking risk groups at random from the set of all network risk groups, and inputing the union of all IP links that are associated with these risk groups to the SCORE algorithm. We evaluated the accuracy of the algorithm in terms of the fraction of faults correctly localized by the algorithm.

As a baseline experiment (not shown), we measure the accuracy of SCORE as a function of the number of simultaneous faults for different types of SRLGs (ports, modules, etc.). The accuracy of the algorithm on these data sets is greater than 95\% for all types of risk groups for fewer than five simultaneous failures. For failure scenarios involving only a single fiber cut, router failure or module failure, which form the common case for hard failures, our simulation results indicate the accuracy is near $100 \%$. These high accuracy numbers are expected since there are no imperfections; SCORE outputs a wrong hypothesis only when fault signatures from two different faults combine to produce another fault's signature, which is typically rare.

2) Imperfect fault notifications: Next, we simulate imperfections due to operational realities, such as the loss of failure notifications. We consider three parameters: the error threshold used in the SCORE algorithm, the number of simultaneous failures, and the loss probability (which represents the percentage of IP link failure notifications lost for a given failure scenario).

Figures 4(a) and 4(b) demonstrate the accuracy of the algorithm under a range of loss probabilities for different numbers of simultaneous failures and error thresholds. Specifically, the figures plot the percentage of correct hypotheses as a function of the error probability. In Figure 4(a) the algorithm error threshold is fixed at 0.6 and the number of simultaneous failures is varied from 1 to 5 . In Figure 4(b), the algorithm error threshold is varied from 0.6 to 1.0 , while the number of simultaneous failures is set to 3 . As expected, increasing the loss probability reduces the accuracy of the algorithm. Under three simultaneous failure events and an error probability of 0.1, we can observe from Figure 4(b) that an algorithm error threshold between 0.7 and 0.8 restores the accuracy of the SCORE algorithm to around $90 \%$. However, if we mandate 


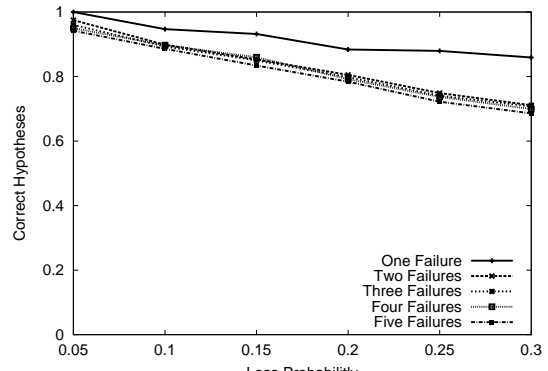

(a) Fixed error threshold of 0.6

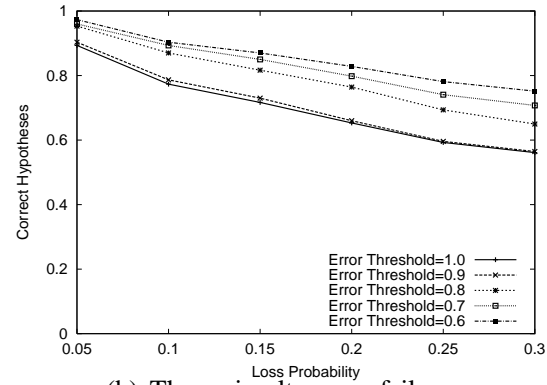

(b) Three simultaneous failures

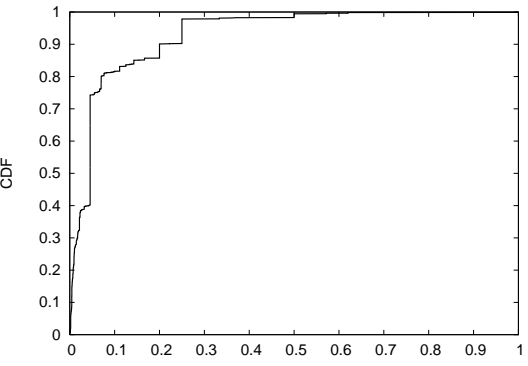

(c) Localization efficiency on real failure data

Fig. 4. The sub-plot (a) shows the accuracy of the SCORE algorithm with a fixed error threshold of 0.6 with a varying number of simultaneous failures as a function of observation loss. Sub-plot (b) illustrates the impact of different error thresholds. Sub-plot (c) shows the localization efficiency defined in Section V-B.3.b over 3000 real failure events.

perfect matching of failure observations to SRLGs (i.e., error threshold $=1.0$ ), then our accuracy in isolating our fault drops to around $78 \%$. This shows the necessity and effectiveness of the error thresholds introduced into the algorithm for fault localization in the face of noisy event observation data.

3) Experience with real failure data: The LINK FAULT system has been operating in a tier-1 backbone network in an off-line fashion to localize IP link failures reported in the network for more than a year. The implemented system operates on a range of fault and performance data, including IP fault notifications and optical-layer performance measures. However, we limit our discussion here to our experience with link failure events.

a) Manual analysis of real failures: Determining whether or not the system correctly localized a given fault requires identification of the root cause of the fault via other means. In many cases, identifying this root cause involves sifting through large amounts of data and reports that is potentially tedious. We therefore selected a set of 18 faults for which we identified the root cause of the problem and compared with the hypothesis output by the system.

Overall, we were able to verify manually that SCORE successfully localized all of the 18 faults studied to the failed network elements (shown in Table I). However, when we used a threshold of 1.0 (i.e., mandated that an SRLG can be identified if and only if faults were observed on all corresponding IP links), we were typically unsuccessful-particularly for router failures, and for the protocol bug reported. In the majority of the router failures, even though these events corresponded to routers being rebooted, the remote ends of the links terminating on these routers did not always report associated link-level events. This may be due to a number of possible scenarios - the events may never have been logged in the syslogs, data may have been lost from the syslogs, the links may have been operationally shut down and, hence, did not fail at this point in time, or the links were not affected by the reboot. Independent of why the link notifications were not always observed, the router failures were all successfully localized when the threshold was marginally reduced. This highlights the importance of the threshold concept in the SCORE algorithm to localize faults in operational networks.

We studied four SONET network element failures. The first_an optical amplifier failure-induced faults on 13 IP links. With a threshold of 1.0 our algorithm identified eight different SRLGs as being involved in the hypothesis. However, as the threshold was reduced to 0.9, the hypothesis size reduced to only two SRLGs, one of them being the actual failed optical amplifier. Further reductions in this threshold did not reduce the number of SRLGs in the hypothesis. Upon investigation, we found that our SONET database was missing one of the IP links in the failure signature. Thus, the SCORE algorithm was unable to attribute this particular IP link to the SONET SRLG, and instead incorrectly concluded that a router port was also involved (the second SRLG) to explain this individual link. The remaining 12 IP links, however, were successfully attributed to the failed optical amplifier. This example illustrates why lowering the threshold is required when there are errors in the database.

The other three SONET failures were all correctly isolated to the SRLG containing the failed network element; in two cases we again had to lower the threshold used within the algorithm to account for links for which we had no failure notification. In one of these cases, the missing link was indeed a result of the interface having been operationally shut down shortly before the failure. Our topology snapshots are generated on a daily basis; therefore the topology change was not reflected in the risk model. These examples clearly indicate the need to deal with operational issues such as incorrect data and erroneous databases.

On another previously identified failure scenario affected by an SRLG database error (fiber A in Table I), the system was unable to identify a single SRLG as being the culprit even as the threshold was lowered, because no SRLG in the database contained all of the circuits reporting the fault. So again, a database error was highlighted by the system's inability to correlate the failure to a single SRLG.

The final case that we evaluated was one in which a lowlevel protocol implementation problem (software bug) affected a number of links within a common OSPF area. This scenario occurred over an extended period of time, during which three other independent failures were simultaneously observed in other areas. When a threshold of 1.0 was used in the SCORE algorithm, the event in question was identified as being the result of 20 independent SRLG failures-a large number even for the extended period of time. As the threshold was reduced to a final value of 0.7 , the event was isolated to 


\begin{tabular}{|c|c|c|c|c|c|c|}
\hline $\begin{array}{l}\text { Type of } \\
\text { problem }\end{array}$ & $\begin{array}{l}\text { Component } \\
\text { Name }\end{array}$ & $\begin{array}{c}\text { \#SRLGS } \\
\text { (Thld.=1.0) }\end{array}$ & Final Thld & $\begin{array}{c}\text { \#SRLGS } \\
\text { (Thld.=Final) }\end{array}$ & $\begin{array}{l}\text { \#Correct } \\
\text { localized }\end{array}$ & Comment \\
\hline \multicolumn{7}{|c|}{ ROUTER } \\
\hline Router & Router A & 27 & 0.8 & 1 & 1 & No event reported by some links \\
\hline Router & Router B & 20 & 0.9 & 3 & 3 & No event reported by some links \\
\hline Router & Router C & 12 & 0.7 & 1 & 1 & No event reported by some links \\
\hline Router & Router D & 1 & 1 & 1 & 1 & - \\
\hline Router & Router E & 18 & 0.8 & 1 & 1 & No event reported by some links \\
\hline Router & Router F & 1 & 1 & 1 & 1 & - \\
\hline Router & Router G & 4 & 1 & 4 & 4 & One router and three links failed \\
\hline \multicolumn{7}{|c|}{ MODULE } \\
\hline Module & Module A & 1 & 1 & 1 & 1 & - \\
\hline Module & Module B & 1 & 1 & 1 & 1 & - \\
\hline Module & Module C & 1 & 1 & 1 & 1 & - \\
\hline \multicolumn{7}{|c|}{ SONET } \\
\hline$\overline{\mathrm{OA}}$ & Sonet A & 8 & 0.9 & 2 & 1 & $\begin{array}{l}\text { No observation reported by one link and } \\
\text { database problem }\end{array}$ \\
\hline $\begin{array}{l}\text { Failed } \\
\text { Transceiver }\end{array}$ & Sonet B & 1 & 1 & 1 & 1 & - \\
\hline $\begin{array}{l}\text { Short } \quad \text { term } \\
\text { Flap }\end{array}$ & Sonet C & 2 & 0.7 & 1 & 1 & No observation reported by one link \\
\hline $\mathrm{OA}$ & Sonet D & 2 & 0.6 & 1 & 1 & No observation reported by one link \\
\hline \multicolumn{7}{|c|}{ FIBER } \\
\hline Fiber Cut & Fiber A & 3 & 0.5 & 1 & 1 & Database problem \\
\hline Fiber Span & Fiber Span A & 1 & 1 & 1 & 1 & - \\
\hline \multicolumn{7}{|c|}{ PROTOCOL } \\
\hline Protocol Bug & OSPF Area A & 20 & 0.7 & 4 & 4 & Incorrect SRLG modelling \\
\hline Protocol Bug & OSPF Area A & 4 & 1 & 4 & 4 & OSPF Area A MPLS enabled interfaces \\
\hline
\end{tabular}

TABLE I

SUMMARY OF 18 ACTUAL, DIAGNOSED FAILURES IN A TIER-1 ISP.

four individual SRLGs: three SRLGs in other OSPF areas (corresponding to the independent failures) and the OSPF area in question. Thus, the SCORE algorithm was correctly able to identify that the event corresponded to a common OSPF area. However, further investigation uncovered that the reason why not all links in the OSPF area were affected was that only those interfaces that were currently MPLS-enabled were affected. Thus, an additional SRLG was added to our SRLG database that incorporated the links in a given area that were MPLS-enabled; application of this enhanced SRLG database successfully localized all of the SRLGs affected by the four simultaneous failures with a threshold of 1.0.

b) Localization efficiency: Figure 4(c) shows the cumulative distribution function of the localization efficiency achieved by our system on 3,000 faults experienced in a tier-1 ISP. Our system appears able to localize faults to less than $5 \%$ for more than $40 \%$ of the failures and to less than $10 \%$ for more than $80 \%$ of the failures. This clearly demonstrates that the SCORE algorithm can efficiently ferret out likely causes from of a large set of possible causes for a given failure. Unfortunately, due to the extensive manual labor involved in diagnosing failures, we do not know the true cause of all 3,000 failures and cannot measure accuracy on this dataset.

\section{Evaluation results for PATH FAULT}

Similar to the previous subsection, we use both simulation and offline analysis of real failure data to evaluate the ability of a system based on MAX-COVERAGE to address the PATH FAULT scenario.

We built a simulator that can inject artificial failures that mimic real-life failure scenarios, obtain observations corresponding to the failure, and then apply localization algorithm to evaluate the accuracy. As described earlier, the entire failure signature is generally not available for fault localization. Therefore, for these simulations, we vary the fraction of the failure signature and compare the accuracy and precision of the localization algorithm. The fraction of the signature $\alpha$ is directly related to the duration of the failure and the rate at which probes are issued. We simulate a fault detection system that issues periodic probes from every router at a rate of one per minute. Therefore, any persistent failure that lasts more than a minute should be captured completely by the monitoring system.

For our simulations, we use the same tier-1 network topology for which we present experiences with real failure data later in Section V-C.3. We simulate three different scenarios: without any noise, with random noise, and with structured noise. The scenarios without any noise, while unrealistic, determine an upper bound on the accuracy of the algorithm. Random noise simulates failure scenarios where the failure signature is mixed with spurious probe losses in the network, often due to transient congestion. In our simulations, we added a random number of spurious observations with an average of 80 per failure. Structured noise, on the other hand, models scenarios where failures of short duration overlap with the 


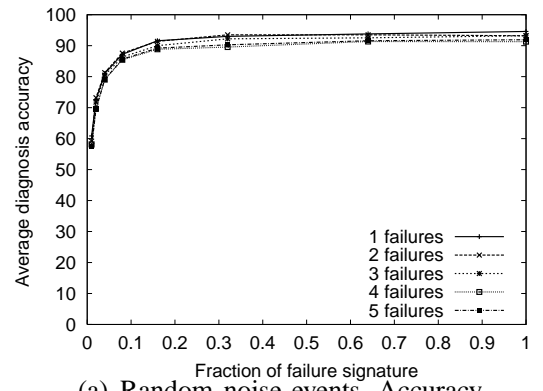

(a) Random noise events, Accuracy

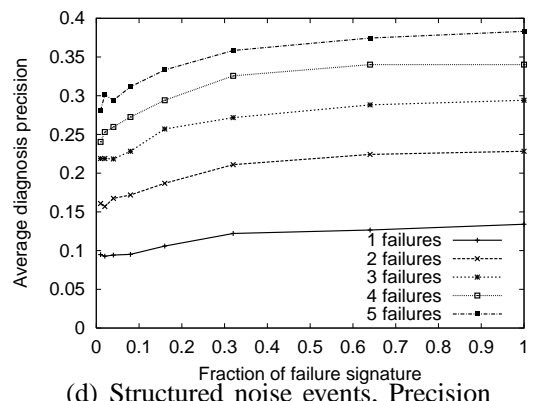

Fraction of failure signature
(d) Structured noise events, Precision

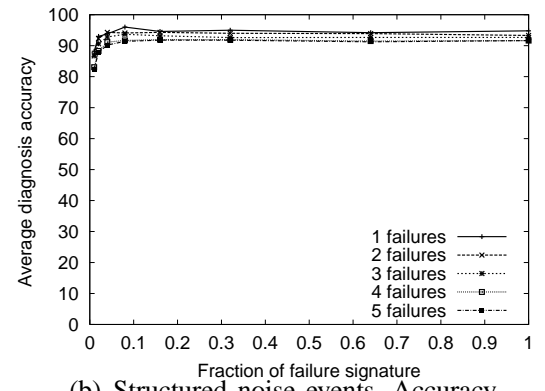

(b) Structured noise events, Accuracy

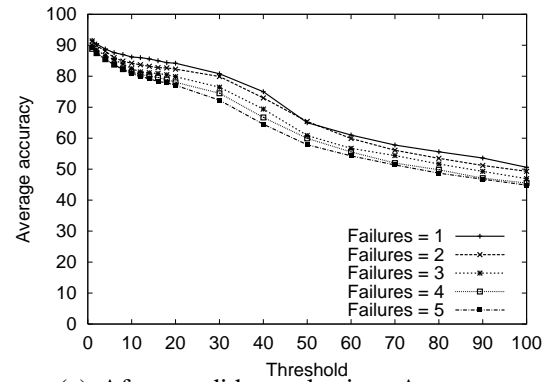

(e) After candidate selection, Accuracy

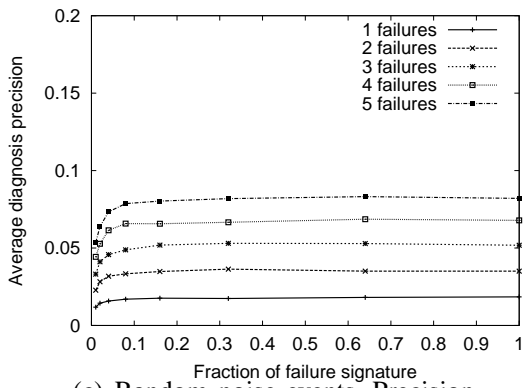

(c) Random noise events, Precision

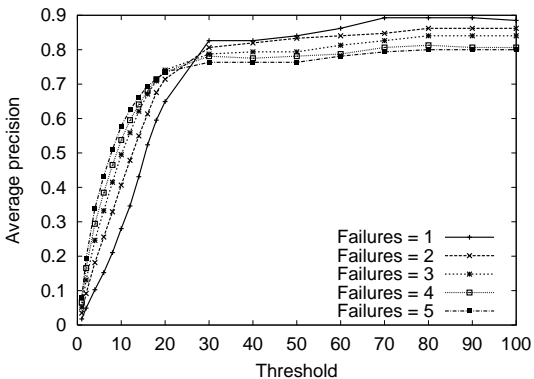

(f) After candidate selection, Precision

Fig. 5. Figure shows the accuracy and precision of MAX-COVERAGE for different numbers of simultaneous failures and when random and structured noise events are injected along with the actual failure. The y-axis is the average accuracy/precision measured over 500 random link failure scenarios, while the $\mathrm{x}$-axis is the fraction of the failure signature. The last two graphs show accuracy and precision after applying the candidate selection algorithm to the output of MAX-COVERAGE for random noise events. For these two graphs, we fixed the signature fraction to 0.16 .

main failure(s) and appear as noise. As an arbitrary starting point, we fail 5 links at random for 5 seconds; the simulated "real" faults last for 60 seconds.

1) Accuracy of the localization algorithm: We measured the average accuracy as a function of the fraction of signature, $\alpha$ for varying number of simultaneous failures and for the three different types of failure scenarios. In simulations with no noise, we observed that the average accuracy is well above $90 \%$ even with five simultaneous failures and only $1 \%$ of the failure signature. Intuitively, this is because the groups of OD-pairs that form the failure signature for each link are large; hence, even a small fraction can create a sample of observations that can uniquely identify the injected failure.

When random noise is introduced into the failure signature (shown in Figure 5(a)), we observed that the accuracy is reduced. In particular, lower fractions of the failure signatures are much more susceptible to noise than the higher ones. For example, at $\alpha=0.01$, the average accuracy is only $60 \%$, while it reaches $90 \%$ at $\alpha=0.16$. This is because at smaller fractions of the failure signature, there is a higher chance that the spurious observations can morph the failure signature of one shared risk into another. Since our algorithm tries to identify risk groups with highest coverage first, it is possible that the failure signature combined with noise will match a candidate risk group other than the injected failure.

With structured noise (in Figure 5(b)), we observe a similar, although less pronounced, phenomenon. The accuracy dips a little compared to the case when there is no noise but is higher than with random noise. The reason is as follows. Since noise is more structured in this case, the resultant failure signature is a composition of $\alpha$ fraction of the original failure signature and $\alpha \times \beta$ fraction of the five noise links, where $\beta$ is the ratio of the failure durations of the noise and the original failure $(\beta$ $=5 / 60$ in our simulations). Since even a small $\alpha$ is enough to achieve high accuracy for five simultaneous failures with no noise, we achieve high accuracy for the structured noise case.

2) Precision of the localization algorithm: Along with the accuracy, we also evaluated the precision of the localization algorithm - the fraction of truth in the hypothesis - with varying signature fraction $\alpha$. Without noise, the algorithm enjoys extremely high precision, especially when $\alpha>0.16$. Precision drops with lower values of $\alpha$ since the failure signature is not strong enough to distinguish between multiple contending risk groups. We also observe that the precision, similar to accuracy, is higher for scenarios with one failure than those with five. Lower accuracy implies that part of the ground truth is not present in the hypothesis, which in turn means that the hypothesis might contain additional candidates not part of the ground truth (i.e., lower precision) to cover all observations, thus leading to lower precision.

In the presence of noise, we only considered the injected faults as part of the ground truth and not the noise itself. Thus the localization precision is expected to be much lower as the algorithm tries to cover all observations including those caused by noise, which in turn leads to a larger hypothesis. We can observe this trend for both the random (in Figure 5(c)) and structured noise (in Figure 5(d)) scenarios. For the structured noise, though, the precision is higher than that of random noise; fewer risk groups are required to cover the small fraction of structured noise introduced.

We also observe that the precision is higher for five failures than one in both noisy scenarios, while the opposite is true without noise. The reason for this is straightforward: Since the amount of added noise remains constant across the varying 
number of simultaneous failures, the number of spurious observations and, therefore, the additional risk groups required to cover them remains similar in all cases. The amount of truth, however, increases linearly with the number of simultaneous failures injected, thereby increasing the overall precision.

Fortunately, we can improve the precision without significantly decreasing the accuracy by applying the candidate selection algorithm described in Section IV-C. In Figures 5(e) and 5(f), we plot the accuracy and precision obtained after applying the ABSOLUTE candidate selection algorithm for different absolute thresholds. For this experiment, we fixed the fraction of the failure signature to 0.16 , still very low.

Eliminating candidate links from the hypothesis that were less than the threshold improves precision significantly until a threshold of about 25, after which the decrease in accuracy out-weighs the additional benefit obtained by increasing the threshold. The optimum threshold will vary depending on the specifics of the topology and fault detection system and, should be derived empirically for a given deployment.

3) Experience with real data: In addition to the simulations, we also collected failure data from a section of a real MPLS-switched tier-I ISP backbone network. The system monitors MPLS tunnels that originate from a subset of edge routers in the backbone, traversing the backbone and finally terminating at other edge routers. Since the MPLS tunnels are established and maintained using the underlying IP topology (through OSPF), any IP-layer failure can impact the MPLS tunnels above the IP layer. The topology consists of a few hundred routers and the probes are transmitted at a periodic rate of one every minute.

The goal of simulation is to stress-test our system; we consider every probe loss as part of the failure signature, including those due to noise. In the production version of the tool, we are mainly interested in characterizing large failures, thus noise can be reduced by considering only those OD-pairs with more than a threshold number of dropped probes.

We compared our hypothesis with ground truth extracted from three data sources: OSPF LSAs, syslogs and SNMP data. During many routing events in the network, the topology is unstable for a short period and probes can get dropped. For such routing incidents, we compare the hypothesis generated by our algorithm with LSAs corresponding to the routing events. In the core backbone network, many IP links (known as composite links [1]) are in fact, logical bundling of many member interfaces, load-balanced by the router. Member interface failures affect only the set of probes traversing that interface after the failure and before the router load-balances again between other members. Since the composite link is active, such failures do not cause OSPF LSAs but appear in router syslogs. In conditions of high link utilization, such as during failures or during maintenance, links can experience heavy packet loss, and therefore, can cause end-to-end probes to get dropped along these links; such congestion events are found in SNMP data.

Note that the ground truth obtained through these data sets is only approximate, as there can be instances when a link failure is reported in the ground truth (using LSAs, syslogs and SNMP data) but the event does not impact traffic forwarding. In these cases, the failure signature will not contain any OD-pairs that are affected by the spurious LSA or syslog message. In such cases, the natural comparison with our hypothesis (namely, requiring that the ground truth be wholly contained in the hypothesis) is obviously unfair. As a relaxation from this strict accuracy metric (which we refer to as ALL), we define a more conservative accuracy metric called ATLEAST_ONE in which accuracy is defined to be 1 if at least one of the links in the ground truth is contained in the hypothesis and 0 otherwise.

a) Candidate selection algorithm: In Figures 6(a) and 6(b), we plot accuracy using both the ALL and ATLEAST_ONE metrics and precision of localization. For this experiment, we picked the hypothesis with best accuracy among those with different topology snapshots. On the x-axis, we vary the cardinality of the failure signature (number of observations) from 50 all the way up to 1000 observations in steps of 50. On the y-axis, the average accuracy/precision corresponding to all failure intervals that have at least $\mathrm{x}$ observations is shown. In effect, these figures show the trend in the accuracy/precision as the failures impact more and more OD-pairs.

Several conclusions can be drawn. First, the number of failure intervals reduces exponentially from about 600 bins with more than 50 observations to about 20 bins with more than 1000 observations (not shown). This is expected, since the number of large failures is typically much smaller than the number of small failures. Overall, we obtained accuracy and precision of about $80 \%$ when considering failures with more than 150 observations. Second, the accuracy and precision of localization increase as the failure size increases initially from 50 to 150 observations. However, it decreases slightly after that but is inconclusive as the number of failure intervals is too small to have statistical significance. Larger failure signatures can indicate one of three things, assuming noise in the network remains the same across all failures. First, the fraction of the failure signature captured could be higher, i.e., the failure lasted for a larger duration. Second, the failure might have affected many OD-pairs in the network, thus the failure occurred on a popular link that lies on many paths. Finally, there could have been many simultaneous failures, the likelihood of which is not insignificant due to router maintenance events. For the first two cases, it is not surprising that our fault localization algorithm performs well, as larger signature fraction means larger accuracy verified using simulations. For the final case, since we use the ATLEAST_ONE metric, there is a strong chance that at least one of the root causes is in our hypothesis. In fact, accuracy using ALL metric is about $40 \%$ less than the ATLEAST_ONE metric, both due to the approximate nature of our ground truth as well as the presence of many simultaneous failures in ground truth.

Third, an ABSOLUTE threshold of 30 that selects candidate links in the hypothesis that cover at least 30 observations seems to represent a good trade-off between accuracy and precision. Below this threshold, the precision is significantly lower while accuracy is only slightly higher. Increasing the candidate selection threshold beyond 30 leads to a marginal decrease in the average accuracy, while precision does not improve any further. 


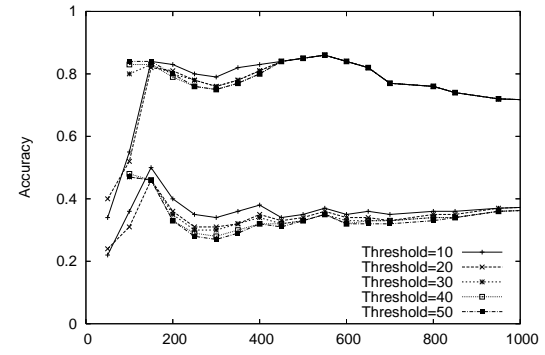

(a) Accuracy for the ATLEA ATservations and ALL (below) metrics, assuming the correct topology is known.

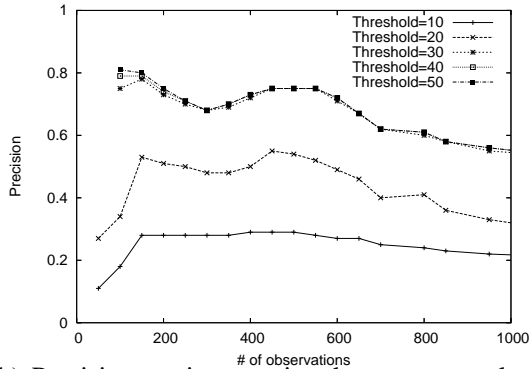

(b) Precision, again assuming the correct topology is known.

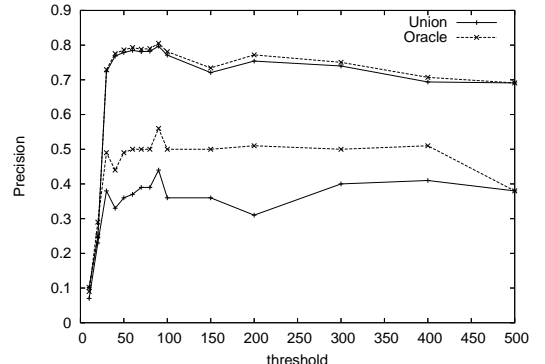

(c) Precision as a function of ABSOLUTE threshold, both assuming the correct topology (above) and using the UNION heuristic (below).

Fig. 6. Accuracy and precision for the MAX_COVERAGE algorithm on real failures from a tier-1 ISP. The first two graphs assume the correct topology snapshot is known, while the third shows the decrease in precision due to the UNION algorithm that considers the superset of the hypotheses obtained using all the topology snapshots during the 15 -minute bin.

b) Hypothesis selection algorithm: In Figure 6(c), we plot the precision for the UNION hypothesis selection algorithm that combines multiple hypotheses obtained using different topology snapshots. The $\mathrm{x}$-axis is the ABSOLUTE candidate selection threshold that we vary from 10 all the way up to 500. For each of these candidate selection thresholds, we identify all those failure intervals that had at least one candidate link remaining in the hypothesis after we apply the candidate selection thresholds and compute the average accuracy/precision for these failure intervals. The number of bins reduces with increasing candidate selection threshold (not shown in the figure) due to the fact that we discard bins that do not have any candidates left in the hypothesis after we apply the threshold.

From Figure 6(c), we can observe that UNION performs similarly to an oracle that can clairvoyantly pick the best out of all the hypotheses generated using different topology snapshots. Because UNION includes the links from in all the hypotheses, it cannot decrease in accuracy according to our definition. As shown by the upper two lines of Figure 6(c), however, precision reduces by a small amount overall. Because the network topology does not change during many of the 15minute bins containing failures, however, Figure 6(c) under reports the impact of not knowing the correct topology. If we consider only the bins that had a change in topology (where UNION has some effect) precision drops about $15 \%$, as shown in bottom two lines of Figure 6(c).

c) Real MPLS black holes: We describe three silent failures we analyzed using our system. In the first incident, misbehavior of a new device that was connected to the periphery of the network caused many routes to go through the device that were then subsequently black-holed. This is a perfect example where we need to consider all the topology changes within a failure interval. In this case, our localization system outputted two candidate links as the hypothesis- the (properly functioning) link before and the (black hole) link after the re-routing of traffic. For this incident, the localization accuracy therefore is $100 \%$ while precision is only $50 \%$.

In another failure scenario, the forwarding component of a line card failed to dequeue packets until the card was reset. Our localization system output a hypothesis that had five candidate links, out of which, when we applied our
ABSOLUTE threshold of 30 eliminated the four false positives out of the hypothesis and contained only the actual failed link. This hypothesis therefore has $100 \%$ accuracy and precision.

Another known black hole scenario happened due to a misconfiguration causing brief loss in connectivity to MPLS paths that traversed that link. Our localization algorithm output a hypothesis that contained four candidate links, two of which were eliminated after we applied our candidate selection algorithm. Out of the remaining two, one was the actual black hole while the other was a false positive. However, the false positive could not be easily distinguished from the actual black hole since both these links appeared on all the paths corresponding to the affected OD-pairs.

\section{DISCUSSION}

In this section, we discuss some of the main lessons learnt and other insights we obtained during our experience.

Not all failures are difficult to localize manually; sometimes, visual inspection may be sufficient. For example, when all failed MPLS tunnels share one end-point, it is easy to isolate the root cause. If the fault lies in the core, however, visual inspection alone is not sufficient as it may require few hours to localize; our system is most appropriate for such situations. Our system is based on passive inference, but one could conceive an 'active diagnosis' mechanism where targeted probes are issued to isolate the failure. The huge administrative overhead (e.g., obtaining per-link measurements is harder compared to end-to-end probes in PATH FAULT) often hinders creating such an active diagnosis framework, however.

Risk model construction is perhaps the most important step of our methodology; it originates from extensive understanding of the failure scenarios. For example, in PATH FAULT, we observed failures are due to topology changes. Thus, our risk model consisted of IP links and not optical layer equipment such as optical amplifiers, and fibers. It is also important to ensure consistency between the risk model and the corresponding failure detection system. For example, in the PATH FAULT, there is no need to model customer facing links in the topology, as the probes never traverse any of those links.

Constructing the right risk model is not easy, even if the category of risks to be modeled was known. For example, in an OSPF network, multiple paths can exist between a given 
source and a destination if the paths share the same cost (ECMP [14]), in which case traffic is split based on a hash function applied on source-destination IP addresses. However, probes measure only one among these multiple paths and it is difficult to know which one they measure. Similarly, in composite links [1], many optical circuits are bundled together into one logical IP link and router splits traffic according to a hash function. Partial failures involving one member circuit can result in the loss of some probes. In these cases, the risk model needs to be constructed based on the instantaneous path traversed by the probe. Since this is difficult, we consider the union of all potential paths, which is not accurate.

It is often not enough to just model the risks once; determining the right risks to model is a continuous process in many cases. For example, in LINK FAULT, modeling an OSPF area as a shared risk was not enough. A failure we observed involved only $70 \%$ of the OSPF area, which we could detect using the error threshold. Upon further investigation, we needed to introduce a new risk - an OSPF area with MPLS enabled in order to capture this failure. Risk models are also almost always dynamic; the rate at which a given risk model exhibits churn varies depending on the problem. Because of this churn, there could be differences between the risk model and reality that may affect localization. Using error thresholds helps mitigate the problem to some extent.

\section{RELATED WORK}

Monitoring and management is a challenging problem for any large network. It is not surprising, then, that a number of research prototypes (e.g., [5], [8], [13], [20], [24], [26]) and commercial products such as NetFACT [15], OpenView [16], IMPACT [17], EXCpert [24], and SMARTS [30] have been developed to provide powerful, generic frameworks for handling fault indicators, particularly diverse SNMP-based [4] measurements and rule-based correlation capabilities. However, their reliance on the inherent monitoring capabilities in the network elements restricts the types of failures these systems can localize. Our systems complement these solutions for the particular problem scenarios described in the paper.

Much of the existing fault-localization literature focuses on generic techniques which have been validated predominantly against simulated data; in this paper, we validate our approach using real failure data from an operational tier-1 ISP network. Our risk-modeling approach follows other approaches studied in the literature that employ dependency graphs for fault localization (see [33] and references therein for a comprehensive survey of such approaches). In many such graphs, dependencies can even be probabilistic in nature, which require statistical inference mechanisms such as Bayesian inference [31] or belief propagation techniques [25], [34]. Unfortunately, these approaches do not typically scale beyond a few nodes (less than 50) in the dependency graphs [25].

One critical component in applying the risk-modeling approach in the LINK FAULT scenario is the construction of SRLGs. Network engineers routinely employ the concept of SRLGs to provision disjoint paths in optical networks, as input into many traffic-engineering mechanisms, and in protocols such as Generalized Multi-Protocol Label Switching (GMPLS). Due to their importance, previous work has attempted to automatically infer SRLGs [28] in the optical domain. To the best of our knowledge, however, we are the first to use SRLGs in combination with higher-layer fault notifications to isolate failures in the optical hardware of a network backbone without the need for physical-layer monitoring.

PATH FAULT problem falls into a general class of inference problems that includes traffic matrix estimation [40], tomography [3], [11], [21], [23], [35], [36], [39], and many others. Hence, techniques applied in these domains could potentially apply. Moreover, the problem of fault isolation is not limited to networking; similar problems exist in any complex system. Regardless of domain, fault detection systems have taken three basic approaches: rule- or model-based reasoning [2], [10], [16], codebook approaches [30], [38], or machine learning (such as Bayesian and belief networks [7], [37], [32]). The difficulty with probabilistic or machine-learning approaches is that they are not prescriptive: it is not clear what sets of scenarios they can handle besides the specific training data. Rule-based and codebook systems (otherwise known as "expert systems") are often even more specific, only being able to diagnose events that are explicitly programmed. Model-based approaches are more general, but require detailed information about the system under test. Dependency-based systems like ours, on the other hand, allow general inference without requiring undue specificity.

\section{CONCLUSIONS}

In this paper, we developed and evaluated a simple yet effective methodology for localization of faults in the network. Our approach based on risk models localizes faults even in the absence of any network-generated alarms, either because they were not available or because the failures were silent in nature, thus aiding network operators in troubleshooting failures even when conventional monitoring fails. While we discuss two specific scenarios in this paper, there may be many other scenarios where our methodology is directly applicable that are yet to be explored. Our extensive evaluation based on both controlled simulation and actual failure data obtained through real-world deployment in a tier-1 ISP, indicates spatial correlation can obtain high localization accuracy and precision in many failure scenarios. In particular, our experience has shown that the full power of statistical models may not be needed in practice: our greedy approximation suffices for the problem scenarios we consider.

\section{REFERENCES}

[1] AVICI Systems Inc., http://www.avici.com

[2] S. Brugbosi, G. Bruno, et al., "An expert system for real-time fault diagnosis of the Italian telecommunications network," in 3rd Symp. on Integrated Network Management, 1993, pp. 617-628.

[3] J. Cao, D. Davis, S. V. Wiel, and B. Yu, "Time-varying network tomography," J. Amer. Statist. Assoc, vol. 95, no. 452, pp. 1063-1075, 2000 .

[4] J. Case, M. Fedor, M. Schoffstall, and J. Davin, "A simple network management protocol (SNMP),” IETF,” RFC 1157, May 1990.

[5] C. S. Chao, D. L. Yang, and A. C. Liu, "An automated fault diagnosis system using hierarchical reasoning and alarm correlation," in Journal of Network and Systems Management, vol. 9, no. 2, 2001, pp. 183-202. 
[6] S. Chaudhuri, G. Hjalmtysson, and J. Yates, "Control of lightpaths in an optical network," Jan. 2000, http://www.research.att.com/areas/ opticalnetworking/IPoverWDMpublications.html.

[7] M. Chen, A. Zheng, J. Lloyd, M. I. Jordan, and E. Brewer, "A statistical learning approach to failure diagnosis," in Int. Conf. on Autonomic Computing, New York, NY, May 2004.

[8] R. H. Deng, A. A. Lazar, and W.Wang, "A probabilistic approach to fault diagnosis in linear lightwave networks," in Integrated Network Management III, Apr. 1993, pp. 697-708.

[9] L. Fang, A. Atlas, F. Chiussi, K. Kompella, and G. Swallow, "LDP failure detection and recovery," IEEE Communications, vol. 42, no. 10, pp. 117-123, Oct. 2004.

[10] G. Forman, M. Jain, M. Mansouri-Samani, J. Martinka, and A. C. Snoeren, "Automated whole-system diagnosis of distributed services using model-based reasoning," in 9th IFIP/IEEE Workshop on Distributed Systems: Operations and Management, Oct. 1998.

[11] A. Gunnar, M. Johansson, and T. Telkamp, "Traffic matrix estimation on a large ip backbone: A comparison on real data," in ACM Internet Measurement Conference, October 2004.

[12] S. Hanks, T. Li, D. Farinacci, and P. Traina, "Generic routing encapsulation over ipv4 networks," IETF," RFC 1702, Oct. 1994.

[13] P. Hong and P. Sen, "Incorporating non-deterministic reasoning in managing heterogeneous network," in Integrated Network Management II, Apr. 1991, pp. 481-492

[14] C. Hopps, "Analysis of an equal-cost multi-path algorithm," IETF," RFC 2992, Nov. 2000.

[15] K. Houck, S. Calo, and A. Finkel, "Towards a practical alarm correlation system," in 4th IEEE/IFIP Symp. on Int. Net. Mgmnt., 1995.

[16] HP Technologies, Open View, http://www.openview.hp.com.

[17] G. Jakobson and M. D. Weissman, "Alarm correlation," IEEE Network, vol. 7, no. 6, pp. 52-59, Nov. 1993.

[18] D. S. Johnson, "Approximation algorithms for combinatorial problems," Journal of Computer and System Sciences, vol. 9, pp. 256-278, 1974.

[19] R. Kompella, J. Yates, A. Greenberg, and A. C. Snoeren, "IP fault localization via risk modeling," in Proc. Networked Systems Design and Implementation, May 2005.

[20] G. Liu, A. K. Mok, and E. J. Yang, "Composite events for network event correlation," in Integrated Network Management VI, Boston, MA, May 1999.

[21] A. Medina, N. Taft, K. Salamatian, S. Bhattacharyya, and C. Diot, "Traffic matrix estimation: Existing techniques and new directions," in ACM SIGCOMM, Pittsburg, USA, August 2002.

[22] J. Moy, “Ospf version 2,” IETF," RFC 2328, Apr. 1998.

[23] A. Nucci, R. Cruz, N. Taft, and C. Diot, "Design of IGP link weights for estimation of traffic matrices," in IEEE Infocom, Hong Kong, March 2004.

[24] Y. A. Nygate, "Event correlation using rule and object based techniques," in Integrated Network Management, pp. 278-289.

[25] J. Pearl, Probabilistic Reasoning in Intelligent Systems: Networks of Plausible Inference. San Mateo, CA: Morgan Kaufmann Publishers, 1988.

[26] P.Wu, R. Bhatnagar, L. Epshtein, M. Bhandaru, and Z. Shi, "Alarm correlation engine (ACE)," in Network Operation and Management Symp., 1998, pp. 733-742.

[27] R. Ramaswami and K. Sivarajan, Optical Networks : A Practical Perspective. Academic Press/Morgan Kaufmann, Feb. 1998.

[28] P. Sebos, J. Yates, D. Rubenstein, and A. Greenberg, "Effectiveness of shared risk link group auto-discovery in optical networks," in Optical Fiber Comm. Conf., Mar. 2002.

[29] A. Shaikh and A. Greenberg, "OSPF monitoring: Architecture, design and deployment experience," in NSDI, Mar. 2004.

[30] SMARTS Inc, "http://www.smarts.com."

[31] M. Steinder and A. Sethi, "Increasing Robustness of Fault localization through Analysis of Lost, Spurious and Positive Symptoms," in IEEE Infocom, 2002.

[32] — , "End-to-end service failure diagnosis using belief networks," in Network Operation and Management Symp., Florence, Italy, Apr. 2002.

[33] — " "A Survey of Fault Localization Techniques in Computer Networks," Science of Computer Programming, Special Edition on Topics in System Administration, vol. 53, no. 2, pp. 165-194, Nov. 2004.

[34] - "Non-deterministic fault localization in communication systems using belief networks," IEEE/ACM Transactions on Networking, vol. 12, no. 5, pp. 809-822, Oct. 2004.

[35] C. Tebaldi and M. West, "Bayesian inference on network traffic using link count data," J. American Statistical Assoc., vol. 93, no. 442, pp. 557-576, 1998 .
[36] Y. Vardi, "Network tomography: estimating source-destination traffic intensities from link data," J. American Statistical Assoc., vol. 91, pp. 365-377, 1996

[37] H. Wietgrefe, K. Tochs, et al., "Using neural networks for alarm correlation in cellular phone networks," in Proc. International Workshop on Applications of Neural Networks in Telecommunciations, 1997.

[38] S. A. Yemini, S. Kliger, E. Mozes, Y. Yemini, and D. Ohsie, "High speed and robust event correlation," in IEEE Communications, vol. 34, no. 5, 1996, pp. 82-90.

[39] Y. Zhang, M. Roughan, N. Duffield, and A. Greenberg, "Fast accurate computation of large-scale ip traffic matrices from link loads," in ACM SIGMETRICS 2003, June 2003.

[40] Y. Zhang, M. Roughan, C. Lund, and D. Donoho, "An informationtheoretic approach to traffic matrix estimation," in ACM SIGCOMM, August 2003 .

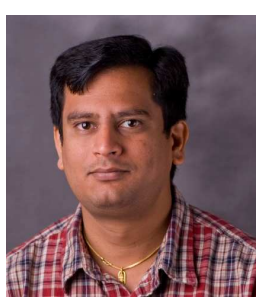

Ramana Rao Kompella (M'07, ACM M'07) is currently an Assistant Professor in the Department of Computer Sciences at Purdue University. His main research interests include fault-management in IP networks, scalable algorithms and architectures for high speed switches and routers, and scheduling in wireless networks. He received his $\mathrm{Ph} . \mathrm{D}$ degree from UCSD in 2007, M.S from Stanford University in 2001, and B.Tech degree from IIT Bombay in 1999.

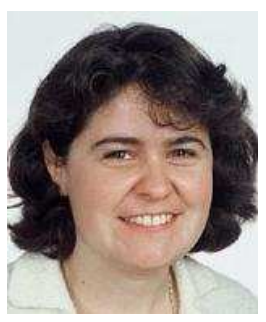

Jennifer Yates is a Technical Specialist in IP Network Management and Performance group at AT\&T Labs - Research. She has worked on IP control of optical networks, and IP network management and performance. She is extremely active both within the research community and within AT\&T - the author of numerous papers, standards contributions, patent applications and an editor of Transactions on Networking. She received her PhD degree from University of Melbourne in 1998.

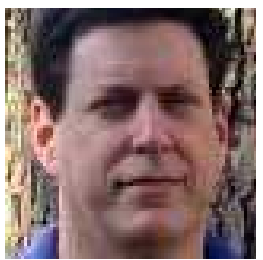

Albert Greenberg is an ACM Fellow, and a Principal Researcher at Microsoft, which he joined in Jan 2007. At Microsoft, he is working on data center networking, enterprise network management, and monitoring. Prior to joining Microsoft, Albert headed the Network Measurement and Engineering Research Dept at AT\&T Labs-Research from 19952006. His research focuses on systems, networking, data centers, and network management. Albert's education includes a BA from Dartmouth College and $\mathrm{MS}$ and a $\mathrm{PhD}$ in Computer Science from the University of Washington $(1981,1983)$.

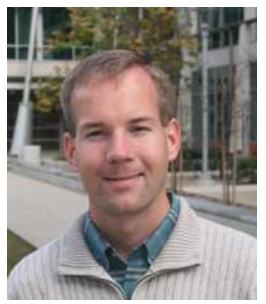

Alex C. Snoeren (S'00, M'03) is an Associate Professor in the Computer Science and Engineering Department at the University of California at San Diego. His research interests include operating systems, distributed computing, and mobile and widearea networking. Prof. Snoeren received the Ph.D. degree in computer science from the Massachusetts Institute of Technology (2003) and the M.S. degree in computer science (1997) and B.S. degree in computer science (1996) and applied mathematics (1997) from the Georgia Institute of Technology, 